\title{
Nonlinear Mapping of Gaussian Statistics: Theory and Applications to Spacecraft Trajectory Design
}

\author{
Ryan S. Park* and Daniel J. Scheeres ${ }^{\dagger}$ \\ University of Michigan, Ann Arbor, Michigan 48109
}

DOI: $10.2514 / 1.20177$

\begin{abstract}
This paper discusses the nonlinear propagation of spacecraft trajectory uncertainties via solutions of the FokkerPlanck equation. We first discuss the solutions of the Fokker-Planck equation for a deterministic system with a Gaussian boundary condition. Next, we derive an analytic expression of a nonlinear trajectory solution using a higher-order Taylor series approach, discuss the region of convergence for the solutions, and apply the result to spacecraft applications. Such applications consist of nonlinear propagation of the mean and covariance matrix, design of statistically correct trajectories, and nonlinear statistical targeting. The two-body and Hill three-body problems are chosen as examples and realistic initial uncertainty models are considered. The results show that the nonlinear map of the trajectory uncertainties can be approximated in an analytic form, and there exists an optimal place to perform a correction maneuver, which is not found using the linear method.
\end{abstract}

\section{Nomenclature}

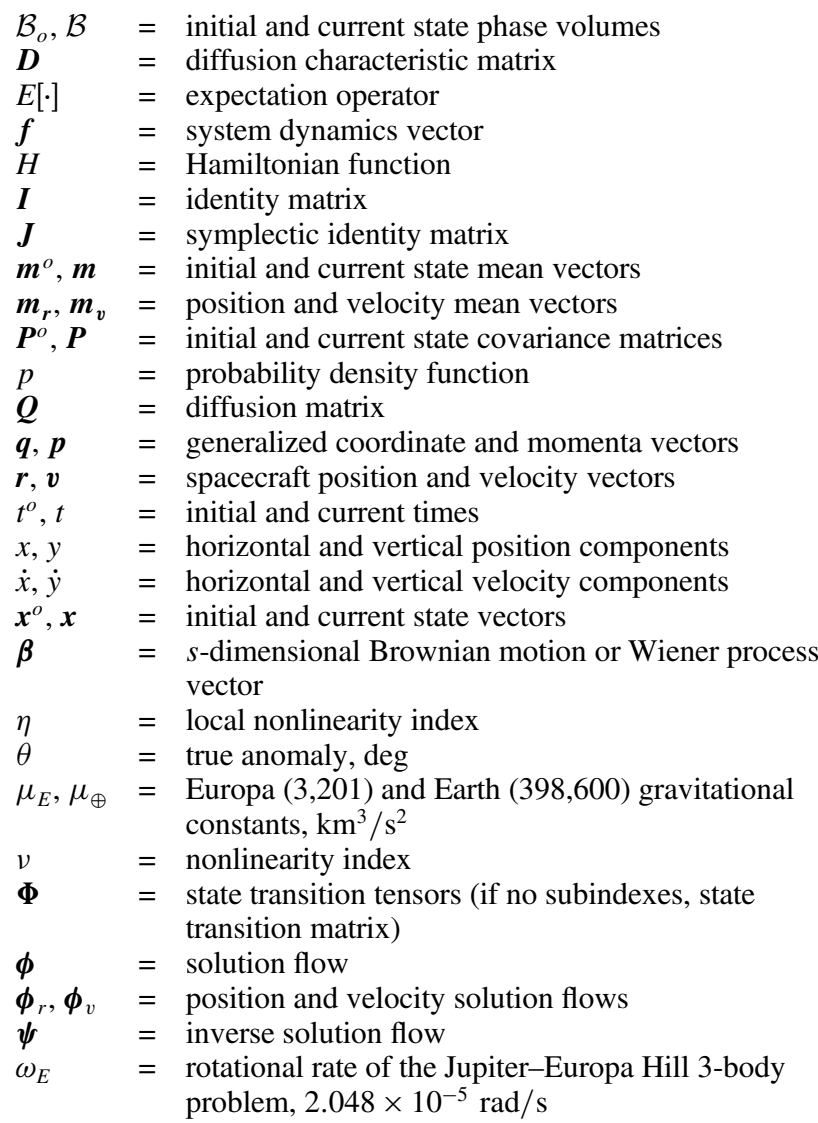

Presented as Paper 05-404 at the 2005 AAS/AIAA Astrodynamics Specialist Conference, Lake Tahoe, California, 7-11 August 2005; received 21 September 2005; revision received 17 January 2006; accepted for publication 6 March 2006. Copyright @ 2006 by the authors.. Published by the American Institute of Aeronautics and Astronautics, Inc., with permission. Copies of this paper may be made for personal or internal use, on condition that the copier pay the $\$ 10.00$ per-copy fee to the Copyright Clearance Center, Inc., 222 Rosewood Drive, Danvers, MA 01923; include the code $\$ 10.00$ in correspondence with the CCC.

${ }^{*}$ Graduate Research Assistant, Department of Aerospace Engineering; sanghp@umich.edu. Member AIAA.

${ }^{\dagger}$ Associate Professor, Department of Aerospace Engineering; scheeres@ umich.edu. Associate Fellow AIAA.

\section{Introduction}

$\mathbf{P}$ RESENT-DAY orbit uncertainty propagation usually chooses between linearized propagation models [1-3] or full nonlinear Monte Carlo simulations [4]. The linear assumption simplifies the problem a great deal; however, the solution fails to characterize trajectory statistics when the system is in a highly unstable environment or when mapped over a long time period. On the other hand, Monte Carlo simulations provide true trajectory statistics, but are computationally intensive and statistics are computed only for a specific epoch and its associated uncertainties. A different approach to orbit uncertainty propagation has also been discussed by Junkins et al. $[5,6]$, in which the effect of the coordinate system on the propagated statistics is thoroughly analyzed; however, the propagation method was based on the linear assumption and the system nonlinearity was not incorporated in the mapping.

In this paper we explore an alternate way to analyze trajectory statistics by incorporating higher-order Taylor series terms that describe localized nonlinear motion, and by solving for the higherorder state solutions as functions of initial conditions, which we call the state transition tensors (STT). Park and Scheeres [7] have shown that the statistics (mean and covariance matrix) computed using the STT approach provide good agreement with Monte Carlo simulations, and we provide more examples using the two-body and Hill three-body problems. As applications of the STTpropagated statistics, we discuss the concept of a statistically correct trajectory, where we analyze the mean trajectory, and its application to nonlinear statistical targeting, where we minimize the expectation of the deviated trajectory. If we solve for a correction maneuver using the STT approach the number of statistical control maneuvers may be reduced because the method captures the system's nonlinearity and provides the most realistic trajectory for the given state uncertainties at the time of the maneuver. We derive necessary conditions required for this problem and present examples to better explain the difference between the linear and nonlinear methods.

\section{Nonlinear Mapping of the System Dynamics}

Consider the spacecraft dynamics governed by the equations of motion as a function of the initial conditions, written in tensor notation:

$$
\dot{\boldsymbol{x}}_{i}(t)=\boldsymbol{f}_{i}[t, \boldsymbol{x}(t)]
$$

where $\boldsymbol{x}=\left\{\boldsymbol{x}_{i} \mid i=1, \ldots, n\right\}$ and initial conditions $\boldsymbol{x}_{i}\left(t^{o}\right)=\boldsymbol{x}_{i}^{o}$. For a given initial condition, we denote the solution flow as

$$
\boldsymbol{x}(t)=\boldsymbol{\phi}\left(t ; \boldsymbol{x}^{o}, t^{o}\right)
$$


which is the mapping of the state at epoch to the state at a future time. The solution flow satisfies

$$
\begin{gathered}
\frac{\mathrm{d} \boldsymbol{\phi}}{\mathrm{d} t}=\boldsymbol{f}\left[t, \boldsymbol{\phi}\left(t ; \boldsymbol{x}^{o}, t^{o}\right)\right] \\
\boldsymbol{\phi}\left(t^{o} ; \boldsymbol{x}^{o}, t^{o}\right)=\boldsymbol{x}\left(t^{o}\right)
\end{gathered}
$$

This is somewhat an obvious statement; however, this notation is convenient when we consider the flow of a region in phase space.

Suppose we are given an initial phase volume $\mathcal{B}_{o}$. The evolution of this phase volume can be stated as

$$
\mathcal{B}(t)=\left\{\boldsymbol{x}(t) \mid \boldsymbol{x}(t)=\boldsymbol{\phi}\left(t ; \boldsymbol{x}^{o}, t^{o}\right), \forall \boldsymbol{x}^{o} \in \mathcal{B}_{o}\right\}
$$

Applying a similar approach the inverse solution flow can be stated as

$$
\boldsymbol{x}^{o}=\boldsymbol{\phi}\left(t^{o} ; \boldsymbol{x}, t\right)
$$

which leads to the identity

$$
\boldsymbol{x}^{o}=\boldsymbol{\phi}\left[t^{o} ; \boldsymbol{\phi}\left(t ; \boldsymbol{x}^{o}, t^{o}\right), t\right]
$$

To distinguish between the direct and inverse solution flows, we use the following notation for the inverse solution flow:

$$
\boldsymbol{x}^{o}=\boldsymbol{\psi}\left(t, \boldsymbol{x} ; t^{o}\right)
$$

where $t$ and $\boldsymbol{x}$ are free variables. We note that the initial conditions of a trajectory can also be considered to be integrals of motion of the system, and hence, $\mathrm{d} \boldsymbol{x}^{o} / \mathrm{d} t=0$ [8]. These notations will be used later to solve the Fokker-Planck equations and to derive the nonlinear representations of the mean and covariance matrix.

Using our definition of the solution flow, the deviation of the current state from a nominal trajectory can be represented as

$$
\delta \boldsymbol{x}(t)=\boldsymbol{\phi}\left(t ; \boldsymbol{x}^{o}+\delta \boldsymbol{x}^{o}, t^{o}\right)-\boldsymbol{\phi}\left(t ; \boldsymbol{x}^{o}, t^{o}\right)
$$

which satisfies the equations of motion

$$
\delta \dot{\boldsymbol{x}}(t)=\boldsymbol{f}\left[t, \boldsymbol{\phi}\left(t ; \boldsymbol{x}^{o}+\delta \boldsymbol{x}^{o}, t^{o}\right)\right]-\boldsymbol{f}\left[t, \boldsymbol{\phi}\left(t ; \boldsymbol{x}^{o}, t^{o}\right)\right]
$$

Now perform a Taylor series expansion of the solutions in terms of the initial deviation $\delta \boldsymbol{x}^{o}$. We carry the expansions to the $m$ th order to find

$$
\begin{aligned}
\delta \boldsymbol{x}_{i}(t) & =\sum_{p=1}^{m} \frac{1}{p !} \boldsymbol{\Phi}_{i, k_{1} \cdots k_{p}} \delta \boldsymbol{x}_{k_{1}}^{o} \cdots \delta \boldsymbol{x}_{k_{p}}^{o} \\
\delta \dot{\boldsymbol{x}}_{i}(t) & =\sum_{p=1}^{m} \frac{1}{p !} \boldsymbol{f}_{i, k_{1} \cdots k_{p}}^{*} \delta \boldsymbol{x}_{k_{1}} \cdots \delta \boldsymbol{x}_{k_{p}}
\end{aligned}
$$

where we use the summation convention, $k_{j} \in\{1, \ldots, n\}$, subscripts $k_{j}$ denote the $k_{j}$ th component of the state vector,

$$
\begin{gathered}
\boldsymbol{\Phi}_{i, k_{1} \cdots k_{p}}=\frac{\partial^{p} \boldsymbol{x}_{i}}{\partial \boldsymbol{x}_{k_{1}}^{o} \cdots \partial \boldsymbol{x}_{k_{p}}^{o}} \\
\boldsymbol{f}_{i, k_{1} \cdots k_{p}}^{*}=\left.\frac{\partial^{p} \boldsymbol{f}_{i}}{\partial \boldsymbol{x}_{k_{1}} \cdots \partial \boldsymbol{x}_{k_{p}}}\right|_{\boldsymbol{x}=\boldsymbol{x}^{*}}
\end{gathered}
$$

and the superscript " $*$ " represent the values computed along a reference (nominal) solution. As an example, the summation convention applied to a second order expansion can be written as

$$
\frac{1}{2} \boldsymbol{\Phi}_{i, k_{1}, k_{2}} \delta \boldsymbol{x}_{k_{1}}^{o} \delta \boldsymbol{x}_{k_{2}}^{o}=\sum_{k_{1}=1}^{n} \sum_{k_{2}=1}^{n} \frac{1}{2} \boldsymbol{\Phi}_{i, k_{1}, k_{2}} \delta \boldsymbol{x}_{k_{1}}^{o} \delta \boldsymbol{x}_{k_{2}}^{o}
$$

From now on, we will call the higher-order partials of the state $\boldsymbol{x}_{i}$ (i.e.,
$\boldsymbol{\Phi}_{i, k_{1} \cdots k_{p}}$ ) the state transition tensors (STTs). These relate deviations in the initial conditions to deviations in the state at some future time. The time derivative of the deviation $\delta \dot{\boldsymbol{x}}_{i}$ can also be obtained by differentiating Eq. (11),

$$
\delta \dot{\boldsymbol{x}}_{i}(t)=\sum_{p=1}^{m} \frac{1}{p !} \dot{\boldsymbol{\Phi}}_{i, k_{1} \cdots k_{p}} \delta \boldsymbol{x}_{k_{1}}^{o} \cdots \delta \boldsymbol{x}_{k_{p}}^{o}
$$

because only the STTs depend on time. In conventional practice researchers usually work with the case $m=1$ (i.e., first-order or linear analysis) where Eqs. (13) and (14) are simply the usual "state transition matrix" and the "linear dynamics matrix," respectively. We later show that when the nonlinearity is strong, including higherorder effects provides superior results as compared with conventional linear analysis.

Now, in order to analyze the deviation of $\delta \boldsymbol{x}$ as an analytic function of the initial deviations, we must solve for the STTs. To obtain differential equations for the STTs, we first substitute Eq. (11) into Eq. (12), which gives the equation of $\delta \dot{\boldsymbol{x}}_{i}$ as a function of the STTs and initial conditions. By equating this with Eq. (16) and balancing terms of the same order in $\delta \boldsymbol{x}^{o}$, we obtain the differential equations for the STTs $\left(\dot{\boldsymbol{\Phi}}_{i, k_{1} \cdots k_{n}}\right)$, where the ODE's up to fourth order deviation are given in Eqs. (17-20).

$$
\dot{\boldsymbol{\Phi}}_{i, a}=\boldsymbol{f}_{i, \alpha}^{*} \boldsymbol{\Phi}_{\alpha, a}
$$

$$
\begin{gathered}
\dot{\boldsymbol{\Phi}}_{i, a b}=\boldsymbol{f}_{i, \alpha}^{*} \boldsymbol{\Phi}_{\alpha, a b}+\boldsymbol{f}_{i, \alpha \beta}^{*} \boldsymbol{\Phi}_{\alpha, a} \boldsymbol{\Phi}_{\beta, b} \\
\dot{\boldsymbol{\Phi}}_{i, a b c}=\boldsymbol{f}_{i, \alpha}^{*} \boldsymbol{\Phi}_{\alpha, a b c}+\boldsymbol{f}_{i, \alpha \beta}^{*}\left(\boldsymbol{\Phi}_{\alpha, a} \boldsymbol{\Phi}_{\beta, b c}+\boldsymbol{\Phi}_{\alpha, a b} \boldsymbol{\Phi}_{\beta, c}+\boldsymbol{\Phi}_{\alpha, a c} \boldsymbol{\Phi}_{\beta, b}\right) \\
+\boldsymbol{f}_{i, \alpha \beta \gamma}^{*} \boldsymbol{\Phi}_{\alpha, a} \boldsymbol{\Phi}_{\beta, b} \boldsymbol{\Phi}_{\gamma, c} \\
\dot{\boldsymbol{\Phi}}_{i, a b c d}=\boldsymbol{f}_{i, \alpha}^{*} \boldsymbol{\Phi}_{\alpha, a b c d}+\boldsymbol{f}_{i, \alpha \beta}^{*}\left(\boldsymbol{\Phi}_{\alpha, a b c} \boldsymbol{\Phi}_{\beta, d}+\boldsymbol{\Phi}_{\alpha, a b d} \boldsymbol{\Phi}_{\beta, c}\right. \\
+\boldsymbol{\Phi}_{\alpha, a c d} \boldsymbol{\Phi}_{\beta, b}+\boldsymbol{\Phi}_{\alpha, a b} \boldsymbol{\Phi}_{\beta, c d}+\boldsymbol{\Phi}_{\alpha, a c} \boldsymbol{\Phi}_{\beta, b d}+\boldsymbol{\Phi}_{\alpha, a d} \boldsymbol{\Phi}_{\beta, b c} \\
\left.+\boldsymbol{\Phi}_{\alpha, a} \boldsymbol{\Phi}_{\beta, b c d}\right)+\boldsymbol{f}_{i, \alpha \beta \gamma}^{*}\left(\boldsymbol{\Phi}_{\alpha, a b} \boldsymbol{\Phi}_{\beta, c} \boldsymbol{\Phi}_{\gamma, d}+\boldsymbol{\Phi}_{\alpha, a c} \boldsymbol{\Phi}_{\beta, b} \boldsymbol{\Phi}_{\gamma, d}\right. \\
+\boldsymbol{\Phi}_{\alpha, a d} \boldsymbol{\Phi}_{\beta, b} \boldsymbol{\Phi}_{\gamma, c}+\boldsymbol{\Phi}_{\alpha, a} \boldsymbol{\Phi}_{\beta, b c} \boldsymbol{\Phi}_{\gamma, d}+\boldsymbol{\Phi}_{\alpha, a} \boldsymbol{\Phi}_{\beta, b d} \boldsymbol{\Phi}_{\gamma, c} \\
\left.+\boldsymbol{\Phi}_{\alpha, a} \boldsymbol{\Phi}_{\beta, b} \boldsymbol{\Phi}_{\gamma, c d}\right)+\boldsymbol{f}_{i, \alpha \beta \gamma \delta}^{*} \boldsymbol{\Phi}_{\alpha, a} \boldsymbol{\Phi}_{\beta, b} \boldsymbol{\Phi}_{\gamma, c} \boldsymbol{\Phi}_{\delta, d}
\end{gathered}
$$

The initial conditions for these STTs are simple, $\boldsymbol{\Phi}_{i, a}^{o}=1$ if $i=a$ and all other initial STTs are initially zero. After solving for the STTs, the higher-order solutions can be computed by adding the deviations to the reference solution, or $\boldsymbol{x}_{i}(t)=\boldsymbol{x}_{i}^{*}(t)+\delta \boldsymbol{x}_{i}(t)$. Since the reference solutions $\boldsymbol{x}_{i}^{*}(t)$ are solved while computing the STTs, $\boldsymbol{x}_{i}(t)$ can now be computed as an analytic function of the initial conditions in the neighborhood of the reference solution.

\section{Convergence of the Higher-Order Solutions}

Given the Taylor series expansion of a solution, Eq. (11), it is not trivial to say what order of solution suffices to represent the local nonlinear motion for a given set of initial deviations. In this section, we discuss a systematic way to find the necessary order of Taylor series that captures the local nonlinear behavior.

The level of nonlinearity (or linearity) of a system's coordinate system can be checked by computing its nonlinearity index $[5,6]$

$$
v\left(t, t^{o}\right) \triangleq \sup _{k=1, \ldots, N} \frac{\left\|\boldsymbol{\Phi}^{k}\left(t, t^{o}\right)-\boldsymbol{\Phi}^{*}\left(t, t^{o}\right)\right\|_{f}}{\left\|\boldsymbol{\Phi}^{*}\left(t, t^{o}\right)\right\|_{f}}
$$

where the superscript $k$ represents that the solutions are computed along neighboring trajectories, $N$ is the number of initial sample points, and $\|\cdot\|_{f}$ represents the Frobenius norm. For a given initial error ellipsoid the neighboring trajectories are chosen from the worstcase initial conditions (e.g., boundary points of the 3- $\sigma$ ellipsoid). The nonlinearity index computes the level of maximum linear deviation from the reference trajectory. However, our focus is more 

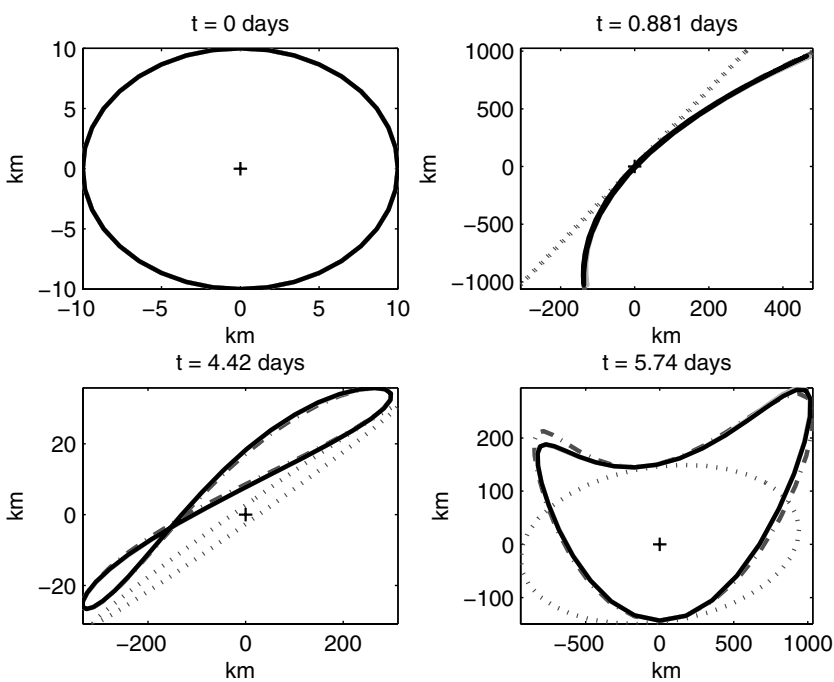

Fig. 1 Phase volume projected onto the position plane, where solid line represents true nonlinear, dotted line represents the 1st order, dashdotted line represents the 2 nd order, and dashed line represents the 3 rd order solutions.

on deciding the sufficient order of the higher-order solution. For this reason, we apply a slightly different approach where we instead propagate the deviated trajectory.

For a fixed future time, the idea is to compute the level of nonlinearity that can be approximated by using the STT approach. Let the local nonlinearity index be defined as follows:

$$
\eta^{m}\left(t, t^{o}\right) \triangleq \sup _{\substack{i=1, \ldots, n \\ k=1, \ldots, N}} \frac{\left|\delta \boldsymbol{x}_{i}^{m}\left(t ; \delta \boldsymbol{x}_{k}^{o}, t^{o}\right)-\delta \boldsymbol{x}_{i}^{*}\left(t ; \delta \boldsymbol{x}_{k}^{o}, t^{o}\right)\right|}{\left|\delta \boldsymbol{x}_{i}^{*}\left(t ; \delta \boldsymbol{x}_{k}^{o}, t^{o}\right)\right|}
$$

where $\delta \boldsymbol{x}_{i}^{m}$ represents a component of the $m$ th-order STT-solution vector [i.e., Eq. (11)], $\delta \boldsymbol{x}_{i}^{*}$ represents a component of the nonlinearly integrated solution vector, and $\delta \boldsymbol{x}_{k}^{o}$ represents the $k$ th sample state vector chosen from the boundary of the initial confidence region (e.g., initial $N-\sigma$ ellipsoid). Note that the subscripts $i \in\{1, \ldots, n\}$ and $k \in\{1, \ldots, N\}$, where $n$ is the system dimension and $N$ is the number of initial sample points. In other words, we find the state that deviates the most from its reference value over all initial samples and each component of the state vector. The computed value of $\eta^{m}$ then tells how well the $m$ th-order solution can approximate the true nonlinear motion. As we consider higher-order Taylor series, and if the series are convergent, $\eta^{m}$ will converge to zero, and by increasing the order of solution we can compute the percent difference between the true and STT solutions. Using this notation, $\eta^{m} \rightarrow 0$ as $m \rightarrow \infty$. As a result, a higher-order solution needs to be considered if $\eta^{m}>\epsilon$, where $\epsilon$ depends on how accurately the user wants to approximate the true nonlinear motion.

As an example, Fig. 1 shows the propagation of a string drawn from the surface of a phase volume and projected onto the position plane [7]. The model is based on the Hill three-body problem that is discussed in the "Example" section. It shows that the higher-order solutions converge to the true nonlinearly integrated trajectory as we consider higher-order solutions. Another important observation is that the level of convergence rate varies along the trajectory, which depends on the system nonlinearity.

\section{Probability Overview}

\section{Review of the Gaussian Distribution}

Consider the spacecraft state vector as a Gaussian random vector (GRV), $\boldsymbol{x} \sim \mathcal{N}(\boldsymbol{m}, \boldsymbol{P})$, where $\boldsymbol{m}$ is the mean vector and $\boldsymbol{P}$ is the covariance matrix. The probability density function for $\boldsymbol{x}$ is defined as

$$
p(\boldsymbol{x})=\frac{1}{\sqrt{(2 \pi)^{n} \operatorname{det} \boldsymbol{P}}} \exp \left[-\frac{1}{2}(\boldsymbol{x}-\boldsymbol{m})^{T} \boldsymbol{P}^{-1}(\boldsymbol{x}-\boldsymbol{m})\right]
$$

An important property of the Gaussian distribution is that the statistics of a GRV can be completely described by the first two moments (i.e., $\boldsymbol{m}$ and $\boldsymbol{P}$ ) using the joint characteristic function (JCF). For a nonzero mean GRV, the first four moments become

$$
\begin{gathered}
E\left[\boldsymbol{x}_{i}\right]=\boldsymbol{m}_{i} \\
E\left[\boldsymbol{x}_{i} \boldsymbol{x}_{j}\right]=\boldsymbol{m}_{i} \boldsymbol{m}_{j}+\boldsymbol{P}_{i j} \\
E\left[\boldsymbol{x}_{i} \boldsymbol{x}_{j} \boldsymbol{x}_{k}\right]=\boldsymbol{m}_{i} \boldsymbol{m}_{j} \boldsymbol{m}_{k}+\left(\boldsymbol{m}_{i} \boldsymbol{P}_{j k}+\boldsymbol{m}_{j} \boldsymbol{P}_{i k}+\boldsymbol{m}_{k} \boldsymbol{P}_{i j}\right) \\
E\left[\boldsymbol{x}_{i} \boldsymbol{x}_{j} \boldsymbol{x}_{k} \boldsymbol{x}_{l}\right]=\boldsymbol{m}_{i} \boldsymbol{m}_{j} \boldsymbol{m}_{k} \boldsymbol{m}_{l}+\left(\boldsymbol{m}_{i} \boldsymbol{m}_{j} \boldsymbol{P}_{k l}+\boldsymbol{m}_{i} \boldsymbol{m}_{k} \boldsymbol{P}_{j l}+\boldsymbol{m}_{j} \boldsymbol{m}_{k} \boldsymbol{P}_{i l}\right. \\
\left.+\boldsymbol{m}_{i} \boldsymbol{m}_{l} \boldsymbol{P}_{j k}+\boldsymbol{m}_{j} \boldsymbol{m}_{l} \boldsymbol{P}_{i k}+\boldsymbol{m}_{k} \boldsymbol{m}_{l} \boldsymbol{P}_{i j}\right)+\boldsymbol{P}_{i j} \boldsymbol{P}_{k l}+\boldsymbol{P}_{i k} \boldsymbol{P}_{j l} \\
+\boldsymbol{P}_{i l} \boldsymbol{P}_{j k}
\end{gathered}
$$

With the use of symbolic manipulators, computing higher Gaussian moments becomes a simple process.

The Gaussian distribution is used widely for astrodynamics applications due to its simplicity and its invariance under linear operations. When we consider mapping a GRV under nonlinear orbital dynamics, we will see that the Gaussian distribution is no longer preserved because $\boldsymbol{x}(t)$ is a nonlinear function of $\boldsymbol{x}^{o}$ in general. However, we can still approximate a non-Gaussian distribution using the first few moments of the variable. This can be checked by comparing the nonlinearly propagated mean and covariance matrix with the Monte Carlo simulation.

\section{Monte Carlo Computations}

The Monte Carlo mean and covariance matrix are computed based on the following equations [9]:

$$
\boldsymbol{m}_{i}(t)=\frac{1}{N} \sum_{k=1}^{N} \boldsymbol{\phi}_{i}\left(t ; \boldsymbol{x}_{k}^{o}, t^{o}\right)
$$

$\boldsymbol{P}_{i j}(t)=\frac{1}{N-1} \sum_{k=1}^{N}\left[\boldsymbol{\phi}_{i}\left(t ; \boldsymbol{x}_{k}^{o}, t^{o}\right)-\boldsymbol{m}_{i}(t)\right]\left[\boldsymbol{\phi}_{j}\left(t ; \boldsymbol{x}_{k}^{o}, t^{o}\right)-\boldsymbol{m}_{j}(t)\right]$

where the subscript $k$ represents the $k$ th sample point that are chosen according to the initial distribution. For example, if we consider a GRV, each sample point is found using the Gaussian random number generator. Based on the law of large numbers and convergence of the statistics, Eqs. (28) and (29) become the true mean and true covariance matrix as we consider more sample trajectories (i.e., $N \rightarrow \infty)$. Precision orbit prediction often relies on Monte Carlo simulations to predict the future state for nonlinear dynamical situations. However, there are three critical disadvantages when using this approach: a) the number of sample trajectories may grow quite large to obtain convergence of the statistics, b) the simulation needs to be repeated for different initial distributions, and c) it does not provide an analytic frame work. These problems make the Monte Carlo simulation computationally quite intensive and statistics specific. In the next section, we derive a way to avoid these problems by incorporating STT solutions when solving for the mean and covariance matrix at a future time.

\section{Fokker-Planck Equation (Forward Kolmogorov Equation)}

Most orbital dynamics problems involving uncertainty can be written using the Itô stochastic differential equation,

$$
d \boldsymbol{x}(t)=\boldsymbol{f}[\boldsymbol{x}(t), t] \mathrm{d} t+\boldsymbol{G}[\boldsymbol{x}(t), t] \mathrm{d} \boldsymbol{\beta}(t)
$$


where $\boldsymbol{G}$ is an $n$-by-s matrix characterizing the diffusion, that is,

$$
\begin{gathered}
E\left[\mathrm{~d} \boldsymbol{\beta}(t) \mathrm{d} \boldsymbol{\beta}^{T}(t)\right]=\boldsymbol{Q}(t) \mathrm{d} t \\
E\left\{\left[\boldsymbol{\beta}\left(t_{2}\right)-\boldsymbol{\beta}\left(t_{1}\right)\right]\left[\boldsymbol{\beta}\left(t_{2}\right)-\boldsymbol{\beta}\left(t_{1}\right)\right]^{T}\right\}=\int_{t_{1}}^{t_{2}} \boldsymbol{Q}(t) \mathrm{d} t
\end{gathered}
$$

As an example, the diffusion vector $\boldsymbol{\beta}$ can be modeled as stochastic acceleration or process noise in case of the spacecraft orbit determination process. The solution to this stochastic differential equation is

$$
\boldsymbol{x}(t)=\boldsymbol{x}^{o}+\int_{t^{o}}^{t} \boldsymbol{f}[\boldsymbol{x}(\tau), \tau] \mathrm{d} \tau+\int_{t^{o}}^{t} \boldsymbol{G}[\boldsymbol{x}(\tau), \tau] \mathrm{d} \boldsymbol{\beta}(\tau)
$$

Systems with deterministic inputs can be simply rewritten by including them in the state vector.

Now let $p(\boldsymbol{x}, t)$ be the probability density function of the stochastic process $\boldsymbol{x}(t)$. Given a system satisfying the Itô stochastic differential equation, the time evolution of the probability density function (pdf) must satisfy the forward Kolmogorov equation (also known as the Fokker-Planck equation) $[4,10]$ :

$$
\begin{aligned}
& \frac{\partial p(\boldsymbol{x}, t)}{\partial t}=-\sum_{i=1}^{n} \frac{\partial}{\partial \boldsymbol{x}_{i}}\left[p(\boldsymbol{x}, t) \boldsymbol{f}_{i}(\boldsymbol{x}, t)\right] \\
& \quad+\frac{1}{2} \sum_{i=1}^{n} \sum_{j=1}^{n} \frac{\partial^{2}}{\partial \boldsymbol{x}_{i} \partial \boldsymbol{x}_{j}}\left\{p(\boldsymbol{x}, t)\left[\boldsymbol{G}(\boldsymbol{x}, t) \boldsymbol{Q}(t) \boldsymbol{G}^{T}(\boldsymbol{x}, t)\right]_{i j}\right\}
\end{aligned}
$$

where the subscript $i$ represents the $i$ th components of a vector and the index $i j$ represents the $(i, j)$ component of a matrix. In the current analysis we will consider a system without diffusion terms, resulting in a simplified form of the equations

$$
\frac{\partial p(\boldsymbol{x}, t)}{\partial t}=-\sum_{i=1}^{n} \frac{\partial}{\partial \boldsymbol{x}_{i}}\left[p(\boldsymbol{x}, t) \boldsymbol{f}_{i}(\boldsymbol{x}, t)\right]
$$

\section{Integral Invariance of Probability}

Consider a dynamical system $\boldsymbol{f}(\boldsymbol{x}, t)$ and let $I$ be an integral of a vector field $M(\boldsymbol{x}, t)$ over some volume $\mathcal{V}$

$$
I=\int_{\mathcal{V}} M(x, t) \mathrm{d} \boldsymbol{x}
$$

The integral $I$ is called an integral invariant if its total time derivative is constant (i.e., $\mathrm{d} I / \mathrm{d} t=0$ ). The sufficient condition for integral invariance is [8]

$$
\frac{\partial M(\boldsymbol{x}, t)}{\partial t}=-\sum_{i=1}^{n} \frac{\partial}{\partial \boldsymbol{x}_{i}}\left[M(\boldsymbol{x}, t) \boldsymbol{f}_{i}(\boldsymbol{x}, t)\right]
$$

and we note that this is identical to the Fokker-Planck equations without diffusion terms. The probability of the state in some phase volume $\mathcal{B}$ can be computed by integrating the pdf

$$
\operatorname{Pr}(\boldsymbol{x} \in \mathcal{B})=\int_{\mathcal{B}} p(\boldsymbol{x}, t) \mathrm{d} \boldsymbol{x}
$$

We have that $p(\boldsymbol{x}, t)$ satisfies the Fokker-Planck equation of a system with no diffusion terms, Eq. (35), and that this equation is equivalent to the sufficiency condition for the probability to be an integral invariant. Hence, the probability of any dynamical system with no diffusion term is an integral invariant [11]. This result has been discussed by Scheeres et al. [12] and combined with Gromov's nonsqueezing theory to derive a new set of constraints that exist for orbit uncertainty propagation.

\section{On the Relation of the Phase Volume and Probability}

Suppose we are given an initial $N-\sigma$ ellipsoid $\mathcal{B}_{o}$ of the initial state $\boldsymbol{x}^{o}$, that is,

$$
\mathcal{B}_{o}=\left\{\boldsymbol{x}\left(t^{o}\right) \mid \boldsymbol{x}^{T}\left(t^{o}\right) \boldsymbol{P}^{-1}\left(t^{o}\right) \boldsymbol{x}\left(t^{o}\right) \leq N^{2}\right\}
$$

As the probability of a deterministic system is an integral invariant, $\operatorname{Pr}[\boldsymbol{x}(t) \in \mathcal{B}]$ should remain unchanged, which indicates that the propagated $\mathcal{B}_{o}$ (i.e., $\mathcal{B}$ ) is an $N-\sigma$ surface (note that the name surface is given because its shape is no longer ellipsoidal because the system dynamics are nonlinear in general). This implies that the confidence region of the current state can be defined by nonlinearly mapping the initial phase volume $\left(\mathcal{B}_{o}\right)$. This approach is, however, a computationally intensive process because hundreds or thousands of points from the surface of $\mathcal{B}_{o}$ must be integrated using the true nonlinear dynamics. For this reason, we usually work with the simple linear model at a penalty of ignoring the higher-order effects. However, once we have the time solution of the STTs, computing the phase volume incorporating the higher-order effects becomes a simple algebraic manipulation, which provides a more accurate solution than the linear case. Considering a Hamiltonian (or Lagrangian) system and the uniqueness of solutions, the outer boundary points of an initial phase volume must map to the outer boundary points of the phase volume computed at a later time. Hence we only need to analyze the behavior of the surface of this $2 n$ dimensional object. After the outer boundary points of $\mathcal{B}_{o}$ are mapped forward in time using the STTs, they can be projected onto the position and velocity spaces to compute the maximum deviations in these spaces with respect to the reference trajectory.

\section{Solution of the Fokker-Planck Equation for a Deterministic Hamiltonian System}

Consider a Hamiltonian, $H(\boldsymbol{q}, \boldsymbol{p}, t)$, which is a function of an $n$-dimensional generalized coordinate $\boldsymbol{q}$ and $n$-dimensional generalized momentum $\boldsymbol{p}$, hence the system is $2 n$ dimensional. The dynamics equations can be written as

$$
\dot{x}(t)=J H_{x}^{T}
$$

where

$$
\boldsymbol{x}=\left[\begin{array}{ll}
\boldsymbol{q}^{T} & \boldsymbol{p}^{T}
\end{array}\right]^{T}
$$

$H_{\boldsymbol{x}}$ is the row-wise partial derivative of $H$ with respect to $\boldsymbol{x}$, and $\boldsymbol{J}$ is the symplectic matrix

$$
\boldsymbol{J}=\left[\begin{array}{cc}
\mathbf{0} & \boldsymbol{I} \\
-\boldsymbol{I} & \mathbf{0}
\end{array}\right]
$$

Both the identity matrices $\boldsymbol{I}$ and the zero matrices $\mathbf{0}$ are $n \times n$.

Assuming no diffusion in the dynamics (e.g., no stochastic accelerations), the Fokker-Planck equation for the pdf, Eq. (34), simplifies to

$$
\frac{\partial p(\boldsymbol{x}, t)}{\partial t}=-\left[\frac{p(\boldsymbol{x}, t)}{\partial \boldsymbol{x}} \dot{\boldsymbol{x}}+p(\boldsymbol{x}, t) \operatorname{tr}\left(\boldsymbol{J} H_{x x}^{T}\right)\right]
$$

where $\operatorname{tr}(\cdot)$ represents the trace of a matrix. The second term in the right-hand-side of the above equation vanishes because

$$
\operatorname{tr}\left(J H_{x x}^{T}\right)=\operatorname{tr}\left[\begin{array}{cc}
H_{p q} & H_{p p} \\
-H_{q q} & -H_{q p}
\end{array}\right]=0
$$

and reduces the Fokker-Planck equation to

$$
\frac{\mathrm{d} p(\boldsymbol{x}, t)}{\mathrm{d} t}=0
$$

This result is generally true for any system derived from a single potential, and thus, most orbital motion represented in Lagrangian form also satisfy this form of the equation (e.g., Hill 3-body problem, restricted 3-body problem, etc.). This time invariance, in conjunction with Liouville's theorem, provides another proof that the probability over some volume $\mathcal{B}$ [Eq. (38)] is indeed an integral invariant.

Consider $\boldsymbol{x}(t)=\boldsymbol{\phi}\left(t ; \boldsymbol{x}^{o}, t^{o}\right)$ to be the solution flow. From the fundamental theorem of calculus and integral invariance we have 


$$
\begin{gathered}
\operatorname{Pr}(\boldsymbol{x} \in \mathcal{B})=\int_{\mathcal{B}} p(\boldsymbol{x}, t) \mathrm{d} \boldsymbol{x} \\
=\int_{\mathcal{B}_{o}} p\left[\boldsymbol{\phi}\left(t ; \boldsymbol{x}^{o}, t^{o}\right), t\right]\left|\frac{\partial \boldsymbol{x}}{\partial \boldsymbol{x}^{o}}\right| \mathrm{d} \boldsymbol{x}^{o} \\
=\int_{\mathcal{B}_{o}} p\left(\boldsymbol{x}^{o}, t^{o}\right) \mathrm{d} \boldsymbol{x}^{o}
\end{gathered}
$$

which gives

$$
p\left[\boldsymbol{\phi}\left(t ; \boldsymbol{x}^{o}, t^{o}\right), t\right]=p\left(\boldsymbol{x}^{o}, t^{o}\right)\left|\frac{\partial \boldsymbol{x}}{\partial \boldsymbol{x}^{o}}\right|^{-1}
$$

where $|\cdot|$ represents the determinant and we assume it is positive. Equation (48) is true for any dynamical systems, but for a Hamiltonian system, it simplifies further. The mapping from $\boldsymbol{x}^{o}$ to $\boldsymbol{x}$ is a canonical transformation, and thus, $\left|\partial \boldsymbol{x} / \partial \boldsymbol{x}^{o}\right|=1$ for all $t$ according to Liouville's theorem. Hence, the pdf must satisfy

$$
p\left[\boldsymbol{\phi}\left(t ; \boldsymbol{x}^{o}, t^{o}\right), t\right]=p\left(\boldsymbol{x}^{o}, t^{o}\right)
$$

or

$$
p(\boldsymbol{x}, t)=p\left[\boldsymbol{\psi}\left(t, \boldsymbol{x} ; t^{o}\right), t^{o}\right]
$$

In other words, the current state pdf can be characterized by the initial pdf, and vice versa. This means that if the solution is known as a function of initial conditions (i.e., is integrated) and the pdf is known at any one time, it can be found for all time. This result is usually assumed in present-day orbit determination process without discussing it in details. This is, however, an important property that arises from the structure of the Hamiltonian dynamics. We address implications of this result in a later section of this paper.

\section{Solution of the Fokker-Planck Equation for a Hamiltonian System} with a Gaussian Boundary Condition

Let us consider a special case of practical interest. Suppose our system is modeled using the Hamiltonian dynamics (n.b., the result can be generalized for the Lagrangian systems derived from a single potential) and the initial state can be represented as a GRV with mean $\boldsymbol{m}^{o}=\boldsymbol{m}\left(t^{o}\right)$ and covariance matrix $\boldsymbol{P}^{o}=\boldsymbol{P}\left(t^{o}\right)$, which are constants, so that

$$
p\left(\boldsymbol{x}^{o}, t^{o}\right)=\frac{1}{\sqrt{(2 \pi)^{2 n} \operatorname{det} \boldsymbol{P}^{o}}} \exp \left\{-\frac{1}{2}\left(\boldsymbol{x}^{o}-\boldsymbol{m}^{o}\right)^{T} \boldsymbol{\Lambda}^{o}\left(\boldsymbol{x}^{o}-\boldsymbol{m}^{o}\right)\right\}
$$

where $\boldsymbol{\Lambda}^{o}=\boldsymbol{P}\left(t^{o}\right)^{-1}$. Recall that Hamiltonian systems have dimension $2 n$. Using Eq. (50), the current state pdf can be stated as

$$
\begin{aligned}
& p(\boldsymbol{x}, t)=\frac{1}{\sqrt{(2 \pi)^{2 n} \operatorname{det} \boldsymbol{P}^{o}}} \exp \left\{-\frac{1}{2}\left(\boldsymbol{\psi}\left(t, \boldsymbol{x} ; t^{o}\right)-\boldsymbol{m}^{o}\right)^{T} \boldsymbol{\Lambda}^{o}\left(\boldsymbol{\psi}\left(t, \boldsymbol{x} ; t^{o}\right)\right.\right. \\
& \left.\left.-\boldsymbol{m}^{o}\right)\right\}
\end{aligned}
$$

It is easy to show that Eq. (52) satisfies the basic conditions to be a valid pdf, which are that its integral over all space equals unity and that it solves the Fokker-Planck equation:

$$
\begin{gathered}
\int_{\infty} p(\boldsymbol{x}, t) \mathrm{d} \boldsymbol{x}=\int_{\infty} p\left[\boldsymbol{\psi}\left(t, \boldsymbol{x} ; t^{o}\right), t^{o}\right] \mathrm{d} \boldsymbol{x} \\
=\int_{\infty} p\left(\boldsymbol{x}^{o}, t^{o}\right)\left|\frac{\partial \boldsymbol{x}}{\partial \boldsymbol{x}^{o}}\right| \mathrm{d} \boldsymbol{x}^{o} \\
=1
\end{gathered}
$$

where we apply the integral invariance of the pdf and symplectic property of the Hamiltonian system (i.e., $\left|\partial \boldsymbol{x} / \partial \boldsymbol{x}^{o}\right|=1$ ). To prove that this is a valid solution we only need to show that $\mathrm{d} p / \mathrm{d} t=0$. Note that

$$
\frac{\mathrm{d} p(\boldsymbol{x}, t)}{\mathrm{d} t}=\frac{\mathrm{d} p\left[\boldsymbol{\psi}\left(t, \boldsymbol{x} ; t^{o}\right), t^{o}\right]}{\mathrm{d} t}=\frac{\partial p}{\partial \boldsymbol{\psi}} \frac{\mathrm{d} \boldsymbol{\psi}}{\mathrm{d} t}
$$

However $\boldsymbol{\psi}\left(t, \boldsymbol{x} ; t^{o}\right)=\boldsymbol{x}^{o}$ are integrals of motion of our system [8], and hence, their total time derivative is zero. This derivation is particularly of interest because, as we will see later, it provides the evolution of the current state statistics as functions of the initial state and its statistics. An interesting observation that can be made from Eq. (52) is that the peak of the pdf is always located at the propagated initial mean according to the deterministic map [i.e., $\left.\boldsymbol{x}=\boldsymbol{\phi}\left(t ; \boldsymbol{m}^{o}, t^{o}\right)\right]$; however, the solution flow is nonlinear in general, and thus, the current state mean vector is no longer located at the peak of the distribution. It is apparent from Eq. (52) that once the state flow $\boldsymbol{\phi}(t)$ can be represented including the higher-order effects and an analytic expression is obtained as a function of the initial state, the statistical moments of the current state can be obtained that are, by definition, more accurate predictions of the propagated statistics than the linear theory. Obtaining an analytic framework of the statistics propagation is discussed in the next section.

\section{Nonlinear Mapping of the Gaussian Distribution}

Consider computation of the mean. Applying the results from Eqs. (49) and (50) we have the following four equivalent expressions for the mean of the state:

$$
\begin{gathered}
E[\boldsymbol{x}(t)]=\int_{\infty} \boldsymbol{x}(t) p(\boldsymbol{x}, t) \mathrm{d} \boldsymbol{x} \\
=\int_{\infty} \boldsymbol{\phi}\left(t ; \boldsymbol{x}^{o}, t^{o}\right) p\left(\boldsymbol{x}^{o}, t^{o}\right) \mathrm{d} \boldsymbol{x}^{o} \\
=\int_{\infty} \boldsymbol{\phi}\left(t ; \boldsymbol{x}^{o}, t^{o}\right) p\left(\boldsymbol{\phi}\left(t ; \boldsymbol{x}^{o}, t^{o}\right), t\right) \mathrm{d} \boldsymbol{x}^{o} \\
=\int_{\infty} \boldsymbol{x}(t) p\left(\boldsymbol{\psi}\left(t, \boldsymbol{x} ; t^{o}\right), t^{o}\right) \mathrm{d} \boldsymbol{x}
\end{gathered}
$$

We observe that Eq. (58) is suitable for computation of the state uncertainties using the STT formulation because the solution flow can be expanded using the Taylor series and higher moments can be computed using the JCF of the initial Gaussian distribution.

Consider the Gaussian boundary condition for the pdf [Eq. (51)]. Assuming a nonzero mean for the initial state, the pdf for the state $\delta \boldsymbol{x}^{o}$ can be obtained via a linear transformation, $\boldsymbol{x}^{o}=\delta \boldsymbol{x}^{o}+\boldsymbol{m}^{o}-\delta \boldsymbol{m}^{o}$, where $\boldsymbol{m}^{o}$ is the initial mean and $\delta \boldsymbol{m}^{o}$ is the initial mean of the deviation. We note that these variables are constants. Applying the change of variable to the pdf yields

$$
p\left(\delta \boldsymbol{x}^{o}, t^{o}\right)=\frac{1}{\sqrt{(2 \pi)^{n} \operatorname{det} \boldsymbol{P}^{o}}} \exp \left\{-\frac{1}{2}\left(\delta \boldsymbol{x}^{o}-\delta \boldsymbol{m}^{o}\right)^{T} \boldsymbol{\Lambda}^{o}\left(\delta \boldsymbol{x}^{o}-\delta \boldsymbol{m}^{o}\right)\right\}
$$

Since the expectation of the nominal trajectory does not change, by definition, it is easier to instead analyze the statistics of the deviated state. Using the STT notation, the current state mean and covariance matrix are

$$
\begin{gathered}
\delta \boldsymbol{m}_{i}(t)=\sum_{p=1}^{m} \frac{1}{p !} \boldsymbol{\Phi}_{i, k_{1} \cdots k_{p}} E\left[\delta \boldsymbol{x}_{k_{1}}^{o} \cdots \delta \boldsymbol{x}_{k_{p}}^{o}\right] \\
\boldsymbol{P}_{i j}(t)=\left(\sum_{p=1}^{m} \sum_{q=1}^{m} \frac{1}{p ! q !} \boldsymbol{\Phi}_{i, k_{1} \cdots k_{p}} \boldsymbol{\Phi}_{j, l_{1} \cdots l_{q}} E\left[\delta \boldsymbol{x}_{k_{1}}^{o} \cdots \delta \boldsymbol{x}_{k_{p}}^{o} \delta \boldsymbol{x}_{l_{1}}^{o} \cdots \delta \boldsymbol{x}_{l_{q}}^{o}\right]\right) \\
-\delta \boldsymbol{m}_{i}(t) \delta \boldsymbol{m}_{j}(t)
\end{gathered}
$$


where $\left\{k_{j}, l_{j}\right\} \in\{1, \ldots, 2 n\}$. Note that $m=1$ gives the ordinary first-order covariance propagation [11], that is, $\delta \boldsymbol{m}(t)=\boldsymbol{\Phi} \delta \boldsymbol{m}^{o}$ and $\boldsymbol{P}(t)=\boldsymbol{\Phi} \boldsymbol{P}^{o} \boldsymbol{\Phi}^{T}-\delta \boldsymbol{m} \delta \boldsymbol{m}^{T}$, where $\boldsymbol{\Phi}$ is the usual state transition matrix and linearly maps the deviation from $t^{o}$ to $t$.

For the cases where $m>1$ it is apparent from Eq. (63) that we need to compute $2 m$ th-order Gaussian moments, which is, however, a one time operation because we only need to compute the moments of the initial GRV using the JCF. The mean and covariance matrix can thus be obtained as functions of time once we have the time solution of STTs and initial first two moments. This results in the computation of the mean and the covariance matrix to be an algebraic operation. If we consider a zero initial mean, all the odd moments of the initial conditions vanish, which is a property of the Gaussian distribution, and the above equations simplify a great deal. Moreover, it is clear from Eq. (62) that the mean will not be zero indicating that the mean deviates from the reference trajectory, whereas the linear analysis assumes that the mean is the reference trajectory. As an example, for the case where $m=2$ with zero initial mean, the mean and covariance matrix become functions of the initial covariance matrix $\boldsymbol{P}^{o}$, that is,

$$
\begin{gathered}
\delta \boldsymbol{m}_{i}(t)=\frac{1}{2} \boldsymbol{\Phi}_{i, a b} \boldsymbol{P}_{a b}^{o} \\
\boldsymbol{P}_{i j}(t)=\boldsymbol{\Phi}_{i, a} \boldsymbol{\Phi}_{j, \alpha} \boldsymbol{P}_{a \alpha}^{o}-\delta \boldsymbol{m}_{i} \delta \boldsymbol{m}_{j}+\frac{1}{4} \boldsymbol{\Phi}_{i, a b} \boldsymbol{\Phi}_{j, \alpha \beta}\left[\boldsymbol{P}_{a b}^{o} \boldsymbol{P}_{\alpha \beta}^{o}\right. \\
\left.+\boldsymbol{P}_{a \alpha}^{o} \boldsymbol{P}_{b \beta}^{o}+\boldsymbol{P}_{a \beta}^{o} \boldsymbol{P}_{b \alpha}^{o}\right]
\end{gathered}
$$

Considering this fact, updating future measurements with respect to the mean trajectory will provide more accurate information about the true trajectory, and thus, faster convergence of the estimation process may be obtained. This also provides more precise predictions of where the true trajectory is.

In terms of numerical complexity, it generally requires $\sum_{k=0}^{m} n^{k+1}$ number of differential equations to be integrated in order to compute the $m$ th-order STTs, which indicates that the computation time grows exponentially as $m$ increases. We note that the position-dependent partials usually vanish for $m \geq 2$ for both the state space and the phase space, which reduces the number of differential equations significantly. The Monte Carlo simulation, on the other hand, requires one to integrate $n \times N$ equations until the solution converges, where $N$ is the number of samples, and it can be difficult to approximate a sufficient number of samples for convergence. Hence, the number of integrated equations for the STTs may exceed that of the Monte Carlo simulation. However, the importance of the STT-approach comes from the fact that the STTs need be integrated only once while varying the epoch statistics, whereas the Monte Carlo analysis need to be carried out for each set of epoch statistics.

\section{Applications of the STT-Propagated Statistics}

In this section we discuss several spacecraft applications where our nonlinear uncertainty propagation method can be used. We first introduce the concept of statistically correct trajectory targeting where we discuss how to incorporate statistical information into the trajectory design. We then extend this idea and present nonlinear statistical targeting by computing the correction maneuver that gives the statistically correct target position at a desired time.

\section{Concept of the Statistically Correct Trajectory}

Conventional mission design usually relies on the deterministic solution of a boundary value problem and no statistical information is taken into account in the design process. The idea of the statistically correct trajectory is to compensate this deterministic trajectory by incorporating statistical information. Figure 2 shows the concept of the statistically correct trajectory. Suppose the trajectory $\boldsymbol{x}(t)$ deterministically gives the desired target state at $t^{f}$ [i.e., $\boldsymbol{x}\left(t^{f}\right)=$ $\boldsymbol{\phi}\left(t^{f} ; \boldsymbol{x}^{o}, t^{o}\right)=\boldsymbol{x}^{f}$ ]. However, in practice $\boldsymbol{x}^{o}$ is uncertain [e.g., $\left.\boldsymbol{x}^{o} \sim \mathcal{N}\left(\boldsymbol{m}^{o}, \boldsymbol{P}^{o}\right)\right]$, and therefore, the mean trajectory, $\boldsymbol{m}^{f}=$ $E\left[\boldsymbol{\phi}\left(t^{f} ; \boldsymbol{x}^{o}, t^{o}\right)\right]$, deviates from the target state. In other words, the

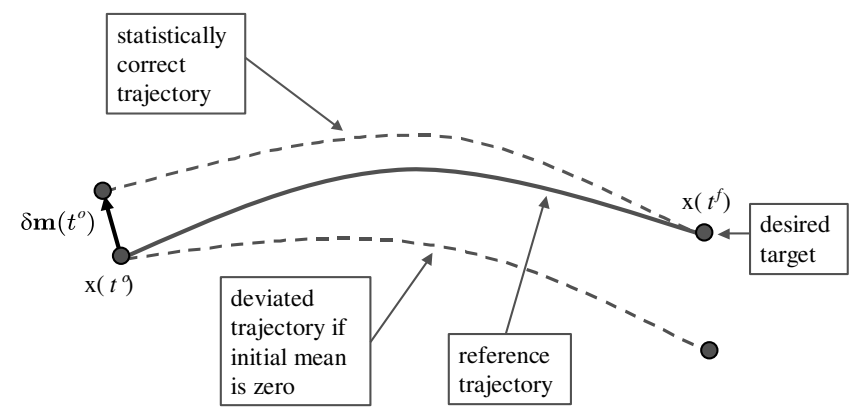

Fig. 2 Illustration of the statistically correction trajectory.

most likely state at time $t^{f}$ is not $\boldsymbol{x}^{f}$, but rather $E\left[\boldsymbol{\phi}\left(t^{f} ; \boldsymbol{x}^{o}, t^{o}\right)\right]=$ $\boldsymbol{m}^{f} \neq \boldsymbol{x}^{f}$ according to probability theory and $\delta \boldsymbol{m}^{f}=\boldsymbol{m}^{f}-\boldsymbol{x}^{f}$ is not zero. Given this fact, and assuming the spacecraft is initially located on the nominal orbit at time $t^{o}$, there exist initial neighboring trajectories $\boldsymbol{x}^{o}+\delta \boldsymbol{m}^{o}$ such that their initial states are offset from the reference state, but their statistical trajectories reach the desired final state at some time $t^{k}$. Mathematically speaking, we want to find $\delta \boldsymbol{m}^{o}$ such that $E\left[\boldsymbol{\phi}\left(t^{k} ; \boldsymbol{x}^{o}+\delta \boldsymbol{m}^{o}, t^{o}\right)\right]=\boldsymbol{x}^{f}$. In general we note that time is a free parameter in this problem (i.e., $t^{k}$ does not have to be $t^{f}$ ), and hence, we solve to minimize the magnitude of $\delta \boldsymbol{m}\left(t^{k}\right)$ with respect to the final state. This is a rather counter intuitive method because we deterministically aim for a different state than the target. We call this trajectory the statistically correct trajectory and the goal is to find $\delta \boldsymbol{m}^{o}$ satisfying $\delta \boldsymbol{m}\left(t^{k}\right)=E\left[\boldsymbol{\phi}\left(t^{k} ; \boldsymbol{x}^{o}+\delta \boldsymbol{m}^{o}, t^{o}\right)\right]-\boldsymbol{x}^{f}=0$.

For an initial Gaussian boundary condition, the mean trajectory can be represented as a power series in the initial mean $\delta \boldsymbol{m}^{\circ}$ and covariance $\boldsymbol{P}^{o}$ using Eq. (62). For example, the 3rd order STTpropagated mean can be analytically stated as

$$
\begin{gathered}
\quad \delta \boldsymbol{m}_{i}\left(t^{k}\right)=\sum_{p=1}^{3} \frac{1}{p !} \boldsymbol{\Phi}_{i, k_{1} \cdots k_{p}} E\left[\delta \boldsymbol{x}_{k_{1}}^{o} \cdots \delta \boldsymbol{x}_{k_{p}}^{o}\right] \\
=\boldsymbol{\Phi}_{i, a} \delta \boldsymbol{m}_{a}^{o}+\frac{1}{2 !} \boldsymbol{\Phi}_{i, a b}\left(\delta \boldsymbol{m}_{a}^{o} \delta \boldsymbol{m}_{b}^{o}+\boldsymbol{P}_{a b}^{o}\right)+\frac{1}{3 !} \boldsymbol{\Phi}_{i, a b c}\left(\delta \boldsymbol{m}_{a}^{o} \delta \boldsymbol{m}_{b}^{o} \delta \boldsymbol{m}_{c}^{o}\right. \\
\left.+\delta \boldsymbol{m}_{a}^{o} \boldsymbol{P}_{b c}^{o}+\delta \boldsymbol{m}_{b}^{o} \boldsymbol{P}_{a c}^{o}+\delta \boldsymbol{m}_{c}^{o} \boldsymbol{P}_{a b}^{o}\right)
\end{gathered}
$$

To solve for the necessary initial state, we must set $\delta \boldsymbol{m}_{i}\left(t^{k}\right)=0$ and solve the resulting equation for the initial mean $\delta \boldsymbol{m}_{i}^{o}$ as a function of time $t^{k}$. The solution to this problem can be found via an iterative process such as Newton's method, that is,

$$
\delta \boldsymbol{m}\left(t^{o}\right)^{i+1}=\delta \boldsymbol{m}\left(t^{o}\right)^{i}-\left.\left\{\frac{\partial\left[\delta \boldsymbol{m}\left(t^{k}\right)\right]}{\partial\left[\delta \boldsymbol{m}\left(t^{o}\right)\right]}\right\}^{-1}\right|_{\delta \boldsymbol{m}=\delta \boldsymbol{m}^{i}} \cdot \delta \boldsymbol{m}\left[t^{k} ; \delta \boldsymbol{m}\left(t^{o}\right)^{i}\right]
$$

with an initial guess of

$$
\delta \boldsymbol{m}\left(t^{o}\right)^{i}=\boldsymbol{\Phi}^{-1} \delta \boldsymbol{m}\left(t^{f}\right)
$$

where we carry out the iteration until the correction is sufficiently small. We note that if we fix the final time (i.e., $t^{k}=t^{f}$ ), a solution exists for the cases with sufficiently small initial uncertainties. After solving for $\delta \boldsymbol{m}^{o}$, the statistically correct initial condition is $\boldsymbol{x}^{o}+\delta \boldsymbol{m}^{o}$ for the given initial covariance matrix. As the orbit covariance becomes small, the solution to the nonlinear problem converges to the solution of the linear problem in Eq. (69). As the initial covariance becomes large, we may require additional terms in the series expansion.

\section{Nonlinear Statistical Targeting}

As an extension of the statistically correct trajectory, we can design a nonlinear statistical correction maneuver using a similar approach. In this paper, we focus on position targeting based on a single impulsive maneuver; however, the result can be generalized to 


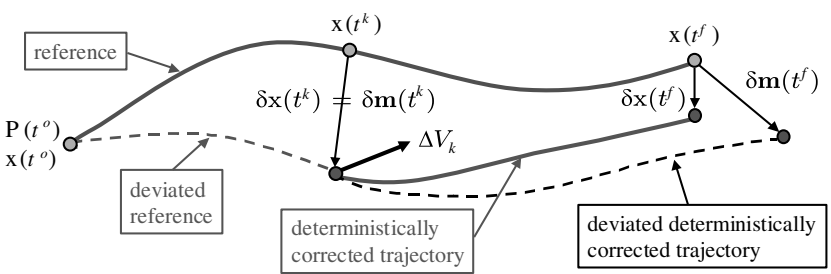

Fig. 3 Illustration of the nonlinear statistical targeting.

target the full state with two or more maneuvers. Figure 3 illustrates the concept of the nonlinear statistical targeting method. In particular, let $\boldsymbol{r}^{f}=\boldsymbol{r}\left(t^{f}\right)$ be the fixed target position and $\boldsymbol{v}^{f}=\boldsymbol{v}\left(t^{f}\right)$ be the velocity vector, which can vary. We want to design a maneuver $\Delta V_{k}$ such that $E\left[\boldsymbol{\phi}_{r}\left(t^{f} ; \boldsymbol{r}^{k}+\delta \boldsymbol{m}_{\boldsymbol{r}}^{k}, \boldsymbol{v}^{k}+\delta \boldsymbol{m}_{\boldsymbol{v}}^{k}+\right.\right.$ $\left.\left.\Delta V_{k}, t^{k}\right)\right]=\boldsymbol{r}^{f}$, where $\boldsymbol{\phi}_{r}$ represents the solution flow of the position components. In other words, we solve for $\Delta V_{k}$ such that $\delta \boldsymbol{m}_{r}\left(t^{f}\right)=0$, which can be stated analytically similar to Eq. (62). We note that $i \in\{1, \ldots, n / 2\}$ because only the position mean is needed.

When we consider the first-order STT (i.e., conventional linear targeting) we are solving for the $\Delta V_{k}$ that satisfies,

$$
\delta \boldsymbol{m}_{\boldsymbol{r}}\left(t^{f}\right)=\boldsymbol{\Phi}_{r r}\left(t^{f}, t^{k}\right) \delta \boldsymbol{m}_{\boldsymbol{r}}\left(t^{k}\right)+\boldsymbol{\Phi}_{r v}\left(t^{f}, t^{k}\right)\left(\delta \boldsymbol{m}_{\boldsymbol{v}}\left(t^{k}\right)+\Delta V_{k}\right)=0
$$

where $\Phi$ 's are the usual state transition matrices mapping the deviation from $t^{k}$ to $t^{f}$. Solving for $\Delta V_{k}$ yields the linear correction maneuver

$$
\Delta V_{k}=-\boldsymbol{\Phi}_{r v}^{-1} \boldsymbol{\Phi}_{r r} \delta \boldsymbol{m}_{\boldsymbol{r}}\left(t^{k}\right)-\delta \boldsymbol{m}_{\boldsymbol{v}}\left(t^{k}\right)
$$

With this linear correction, the deviated position is deterministically solved to be zero [(i.e., $\delta \boldsymbol{r}\left(t^{f}\right)=0$ ]. However, we know that the true trajectory will miss the desired target (depending on the associated uncertainties at $t^{k}$, transit period $t^{f}-t^{k}$, system nonlinearity, etc.) and that this deviation may be significant.

As in the statistically correct trajectory case, the correction maneuver $\Delta V_{k}$ can be computed via an iterative process as in the statistically correct trajectory case. Assuming that the deviated mean is computed using an $m$ th-order STT solution, only one correction maneuver is required to hit the desired target in a statistical sense. In terms of $\Delta V_{k}$ cost, the linear targeting method predicts that it is usually better to perform correction maneuvers early in the trajectory, because the cost is smaller. This yields a tradeoff, however, between the final deviation and the $\Delta V_{k}$ cost (i.e., if a maneuver is performed earlier the statistical trajectory deviates more). Using the nonlinear method, we will always achieve (in a statistical sense) zero for the final deviated position mean, and later examples show that in most cases there is an optimal location (minimum $\Delta V_{k}$ ) to perform the correction maneuver.

\section{Discussion}

Although, in theory, nonlinear statistical targeting provides a maneuver that is more realistic and accurate than the linear theory, there are practical, as well as fundamental, problems that must be discussed before we present results. First we note that the nonlinear statistical targeting depends on statistical knowledge about the epoch state whereas the linear correction maneuver is independent of the statistics, and hence, there is only one maneuver (assumed impulsive) to solve for in the linear targeting problem. The statistical results of the linear maneuver are then checked by making Monte Carlo runs to ensure that the spacecraft resides within the necessary error bound at the target, which is often carried out based on largerthan-estimated initial uncertainties so that a more conservative distribution can be treated at the target. However, we must keep in mind that statistical navigation data are additional sources of information we have about the trajectory, and that the linear correction completely ignores this information in the design process.

If the navigation data are extremely accurate and if the system behaves linearly, both correction maneuvers are essentially the same; however, there are usually significant levels of uncertainties associated at an epoch and the two results will then be different. A practical importance of the nonlinear method is that the number of maneuvers can be reduced when the nonlinear correction is used because the spacecraft will more likely lie within the necessary confidence region for a longer period. An obvious question one may ask is, what if the navigation data are perceived to be too accurate, so that the uncertainties were doubled (e.g., $1-\sigma$ to $2-\sigma$ ), or there are other initial distributions given from different navigation sources. When the uncertainties are increased or changed, the linear maneuver still needs to be checked using the Monte Carlo simulation. This indicates that both methods are, in a way, using the navigation data, but the nonlinear statistical targeting includes the navigation data in the maneuver design process. If there are initial distributions from different navigation sources, it will be the maneuver designer's decision to choose which navigation data are to be used.

\section{Examples}

In this section, we present several examples based on the two-body and Hill three-body body problems. The two-body motion is based on an Earth-to-Moon Hohmann transfer and the Hill three-body model is on the Jupiter-Europa system.

\section{Two-Body Problem}

The governing equations of motion for the planar two-body problem [13] are given as

$$
\begin{aligned}
& \ddot{x}=-\frac{\mu_{\oplus}}{r^{3}} x \\
& \ddot{y}=-\frac{\mu_{\oplus}}{r^{3}} y
\end{aligned}
$$

Figure 4 shows a Hohmann transfer from near Earth $(20,000 \mathrm{~km})$ to the moon $(384,400 \mathrm{~km})$. The orbit is propagated for the transfer period ( $\sim 5.24$ days $)$ and the initial statistics are assumed to be zero mean with position uncertainty of $100 \mathrm{~km}$ and velocity uncertainty of $0.1 \mathrm{~m} / \mathrm{s}$. When computing $\eta$, eight sample points corresponding to the eigenvectors of the initial error ellipsoid are considered. Table 1 shows the local nonlinearity index. The result shows that the series is convergent and the second order solution provides results superior to the first-order case, as predicted by the local nonlinearity index. Figure 5 shows the propagated mean and 1- $\sigma$ ellipsoid plotted with respect to the target state (i.e., apoapsis) where the Monte Carlo result is based on the ensemble of $10^{6}$ sample points. The result shows that the second and higher-order solutions provide a far more accurate estimate of the mean and the dispersion of the samples (i.e., projection of the covariance matrix) than the linear (firstorder) solution.

In this two-body case, we assume that the 3rd order STT solution is considered to be the true Monte Carlo solution, and thus, the $\Delta V_{k}$ computed using the STT method gives the zero deviated position mean. We first solve for the STT solutions of the entire Hohmann

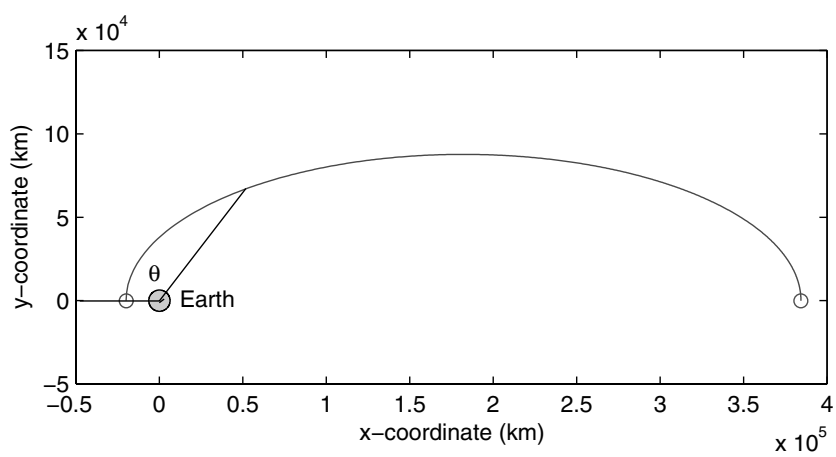

Fig. 4 Two-body Hohmann transfer trajectory. 


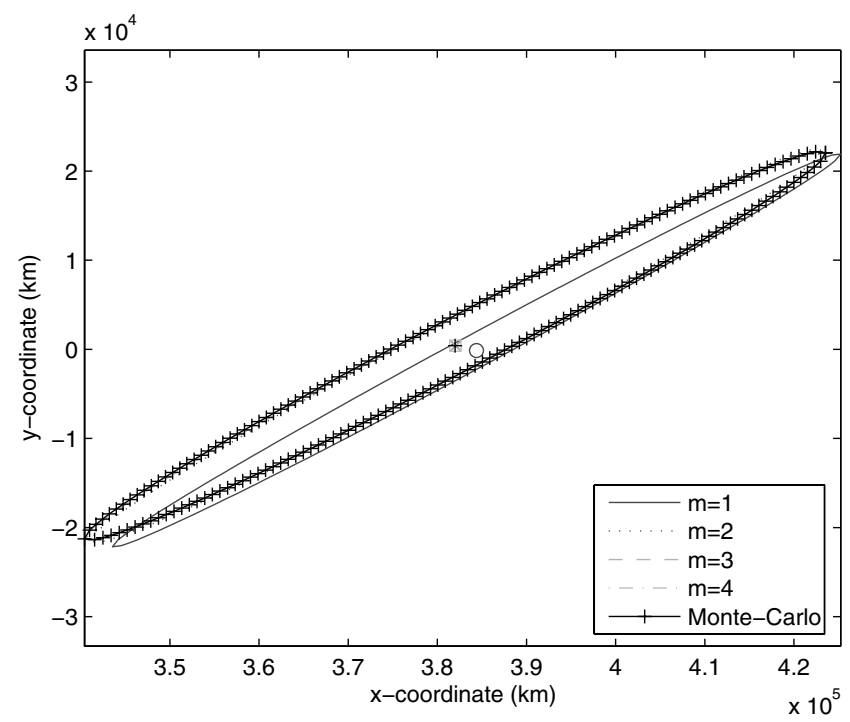

Fig. 5 Comparison of the computed mean and covariance using STT approach and Monte Carlo simulations.

transfer $\left(t^{o}\right.$ to $\left.t^{f}\right)$ and compute the deviated mean at some time $t^{k}$. We then resolve for the STT solutions from the deviated mean at $t^{k}$ to $t^{f}$ and find the correction $\Delta V_{k}$ using Newton's method. There are ways to avoid integrating the STT solutions for each $t_{k}$, such as using the inverse solution of the STTs; however, this is not considered in this study. At every instance of the deviated mean trajectory, the position and velocity uncertainties are assumed to be $100 \mathrm{~km}$ and $0.1 \mathrm{~m} / \mathrm{s}$, respectively, which is the same as the initial uncertainties. The first plot in Fig. 6 shows the $\Delta V_{k}$ corrections computed using the linear and nonlinear methods as function of the true anomaly applied along the deviated mean trajectory. Both solutions become essentially the same after $\theta \approx 90 \mathrm{deg}$; indicating small nonlinearity for the later part of the transfer. As expected, the linear solution $\Delta V_{k}$ grows as the correction maneuver is made at a later time due to a larger deviation in the mean trajectory. We note that there is an optimal (minimum $\left.\left|\Delta V_{k}\right|\right)$ place to perform a correction maneuver that targets the position mean. The second and third plots in Fig. 6 show the deviated position and velocity means at the target. The nonlinear deviated position is not shown because it is zero by definition. When the correction maneuver is made at an early stage of the trajectory using the linear theory, the position mean may deviate quite a bit at the final target. For the deviation in the velocity mean, the difference between the linear and nonlinear solutions is very small.
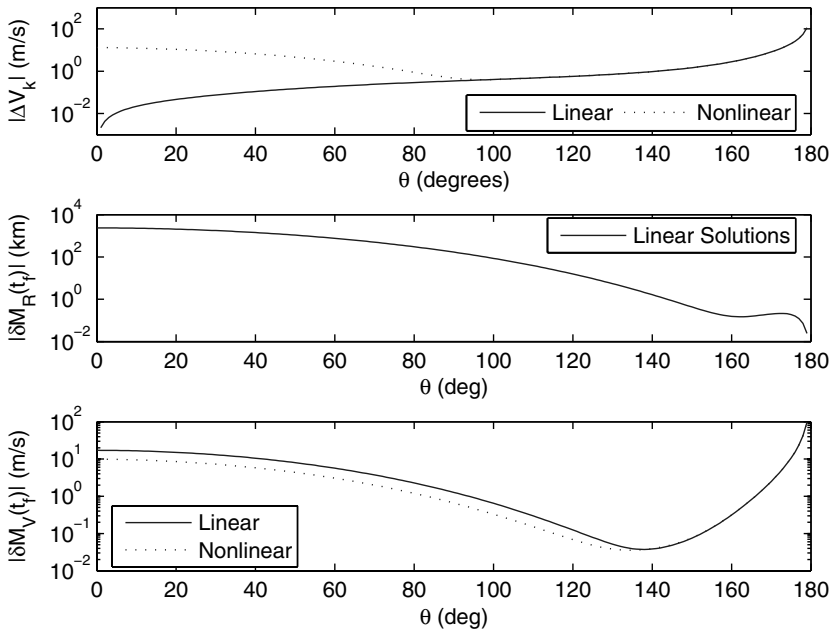

Fig. 6 Computed $\Delta V_{k}$ using the linear and nonlinear methods (first plot) and deviated position and velocity means at the target (second and third plots).

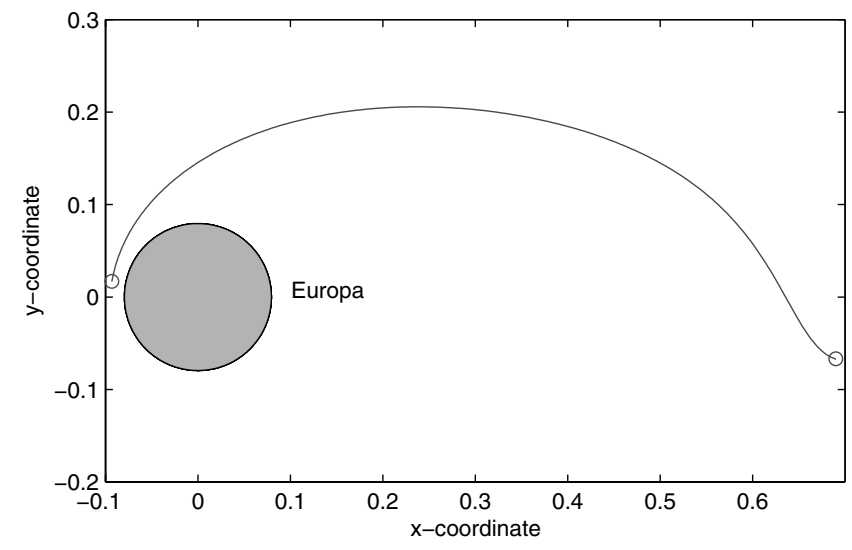

Fig. 7 Hill three-body trajectory.

\section{Hill Three-Body Problem}

The governing equations of motion for the planar Hill three-body problem in nondimensional form are given as [11]

$$
\begin{gathered}
\ddot{x}=2 \dot{y}-\frac{x}{r^{3}}+3 x \\
\ddot{y}=-2 \dot{x}-\frac{y}{r^{3}}
\end{gathered}
$$

The units can be dimensionalized by using the length scale of $\left(\mu_{E} / \omega^{2}\right)^{1 / 3}$ and time scale of $(1 / \omega)$. The reference trajectory in nondimensional coordinates is shown in Fig. 7, where the final position is located at a periapsis. The initial conditions used are

$$
\begin{aligned}
& \boldsymbol{r}\left(t_{o}\right)=\left[\begin{array}{ll}
0.69010031015662 & -0.06716709529872
\end{array}\right] \\
& \boldsymbol{v}\left(t_{o}\right)=\left[\begin{array}{ll}
-0.11045639526249 & 0.03184084790390
\end{array}\right]
\end{aligned}
$$

which is slight below the $L_{2}$ point with Jacobi integral value of -2.15 . Moreover, the initial state is assumed to have a zero mean with position error of $10 \mathrm{~km}\left(5.1 \times 10^{-4}\right.$ in normalized unit $)$ and velocity error of $0.1 \mathrm{~m} / \mathrm{s}\left(2.5 \times 10^{-4}\right.$ in normalized unit). The local nonlinearity index given in Table 1 shows that the fourth order solution provides accuracy better than $10 \%$ error. The level of accuracy can be improved when a different final time is considered because periapsis is the state with the strongest nonlinearity. In Fig. 8, as in the two-body case, the Monte Carlo result is based on an ensemble of $10^{6}$ initial samples. It is clear that the higher-order solution provides a superior result as compared with the linear case. We can see this from the location of the deviated mean and the dispersion of the Monte Carlo samples.

Similar to the two-body case, the 4th order STT-solution is considered to be the true Monte Carlo solution and we assume the mean trajectory has uncertainties that are the same as the initial state, that is, $10 \mathrm{~km}$ for the position components and $0.1 \mathrm{~m} / \mathrm{s}$ for the velocity components. The first plot in Fig. 9 shows the $\Delta V_{k}$ applied at $t^{k}$ that are solved using both the linear and nonlinear methods. The correction maneuvers are plotted as functions of time and we observe small nonlinearity from $t^{k} \approx 15 \mathrm{~h}$ onwards. In this case, however, $\Delta V_{k}$ fluctuates around $t^{k}=4 \mathrm{~h}$ in both cases, unlike the two-body case. This is due to a sudden change in the velocity direction, and hence, the system nonlinearity is varied. There is also an optimal $\left|\Delta V_{k}\right|$, which occurs around $t^{k}=15 \mathrm{~h}$. The second and third plots in

Table 1 Level of higher-order effect

\begin{tabular}{lcc}
\hline \hline$\eta^{m}$ & Two-body & Hill three-body \\
\hline$\eta^{1}$ & 1.06 & 3.57 \\
$\eta^{2}$ & 0.04 & 0.29 \\
$\eta^{3}$ & 0.007 & 0.28 \\
$\eta^{4}$ & 0.001 & 0.06 \\
\hline \hline
\end{tabular}




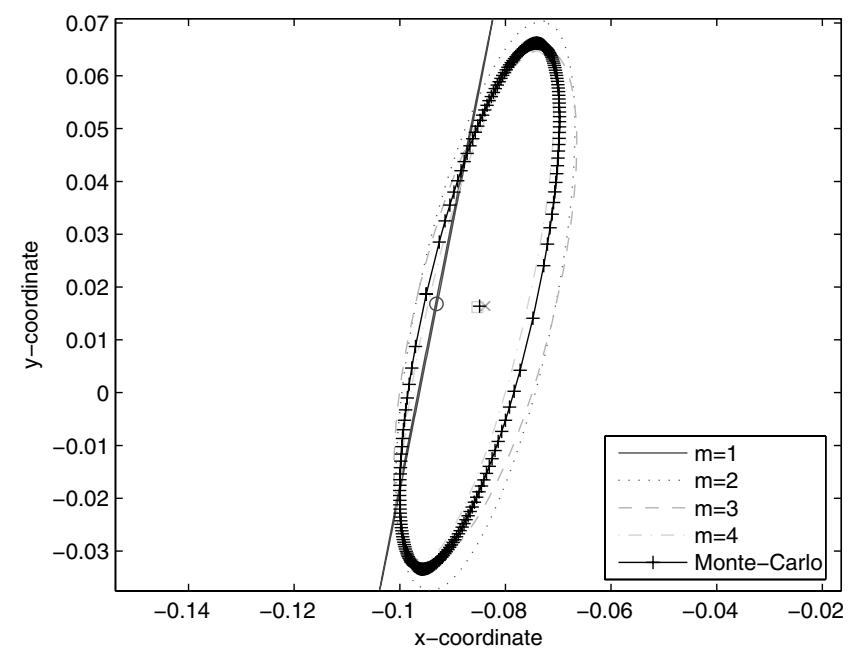

Fig. 8 Comparison of the computed mean and covariance using STT approach and Monte Carlo simulations.
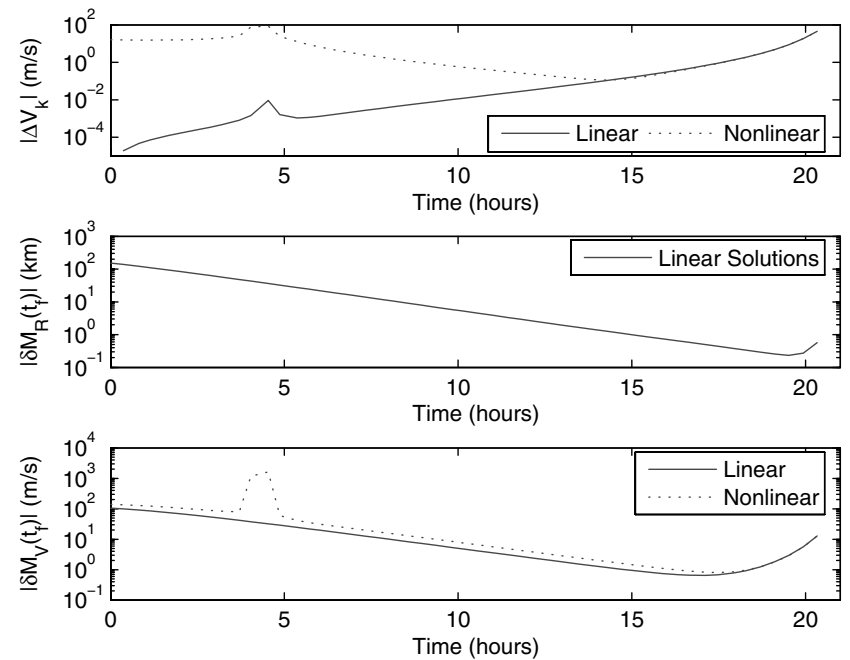

Fig. 9 Computed $\Delta V_{k}$ using the linear and nonlinear methods (first plot) and deviated position and velocity means at the target (second and third plots).

Fig. 9 show the deviated position and velocity means. As in the two-body case, when the correction maneuver is made at an early stage of the trajectory using the linear theory, the position mean may deviate noticeably. The overall difference between the linear and nonlinearly computed velocity mean is very small. A high fluctuation in the nonlinearly solved $\delta \boldsymbol{m}_{v}\left(t^{f}\right)$ is due to the higher correction maneuvers in that time frame.

\section{Discussion}

Several important observations can be made from the previous examples. The first observation is that the linear (first-order) solution captures the semimajor axis of the true covariance plot and the semiminor axis depends on the deviated mean. Second is that the propagated linear mean (i.e., reference state) lies inside the true covariance projection. As a result the nonlinear (or higher order) uncertainty propagation provides a more conservative estimate of the future statistics. This is important because a more realistic error bound can be computed.

\section{Conclusion}

In this study, we have developed an analytic expression of a nonlinear trajectory solution by solving for the higher-order state transition tensors that describe the localized nonlinear motion about a nominal trajectory. We then discussed a fundamental property of the uncertainty propagation by proving the integral invariance of the probability density function via solutions of the Fokker-Planck equations for diffusionless systems. Also presented is the relation between the phase volume and the probability density function, because they actually possess the same statistical information, but are represented in different ways. Applying the nonlinear state propagation and the integral invariance of the probability density function, we have derived an analytic representation of the nonlinear uncertainty propagation and have shown that a sufficient order of state transition tensor approach can be used to replace Monte Carlo simulations with a semianalytical method. This semianalytical method also enables us to introduce the concept of the statistically correct trajectory and its practical application, nonlinear statistical targeting. Nonlinear statistical targeting allows one to use the statistical property of the trajectory in the maneuver design process, and thus, provides a statistically more accurate solution. When the initial uncertainties are negligibly small, the nonlinear method essentially becomes the linear solution; however, when there are sufficiently large initial uncertainties the solution gives the most probable maneuver according to the probability theory. The results from the two-body and Hill three-body examples show that there may exist an optimal place to perform a correction maneuver, a result that is not possible using the linear method.

\section{Acknowledgements}

The research described in this paper was sponsored by the Jupiter Icy Moons Orbiter project through a grant from the Jet Propulsion Laboratory, California Institute of Technology, which is under contract with the National Aeronautics and Space Administration. The authors would like to thank Jon Sims, John Aiello, Lou D'Amario, Ryan Russell, Try Lam, Chris Potts, and Mau Wong from the Jet Propulsion Laboratory for their helpful comments and suggestions.

\section{References}

[1] Battin, R. H., An Introduction to the Mathematics and Methods of Astrodynamics, revised edition, AIAA Education Series, AIAA, Reston, VA, 1999, pp. 623-698.

[2] Montenbruck, O., and Gill, E., Satellite Orbits, 2nd ed., SpringerVerlag, New York, 2001, pp. 257-291.

[3] Crassidis, J. L., and Junkins, J. L., Optimal Estimation of Dynamics Systems, CRC Press LLC, Boca Raton, FL, 2004, pp. 243-410.

[4] Maybeck, P. S., Stochastic Models, Estimation, and Control, Vol. 2, Academic Press, New York, 1982, pp. 159-271.

[5] Junkins, J., Akella, M., and Alfriend, K., "Non-Gaussian Error Propagation in Orbit Mechanics," Journal of the Astronautical Sciences, Vol. 44, No. 4, 1996, pp. 541-563.

[6] Junkins, J., and Singla, P., "How Nonlinear Is It? A Tutorial on Nonlinearity of Orbit and Attitude Dynamics," Journal of the Astronautical Sciences, Vol. 52, No. 1, 2, 2004, pp. 7-60.

[7] Park, R., and Scheeres, D., "Nonlinear Mapping of Gaussian State Covariance and Orbit Uncertainties," AAS Paper 05-170, January, 2005.

[8] Greenwood, D. T., Classical Dynamics, Dover Publications, New York, 1997, pp. 147-271.

[9] Lass, H., and Gottlieb, P., Probability and Statistics, Addison-Wesley, Reading, MA, 1971, pp. 201-229.

[10] Frank, T., Nonlinear Fokker-Planck Equations, Springer-Verlag, New York, 2005, pp. 1-30.

[11] Scheeres, D. J., Han, D., and Hou, Y., "Influence of Unstable Manifolds on Orbit Uncertainty," Journal of Guidance, Control, and Dynamics, Vol. 24, 2001, pp. 573-585.

[12] Scheeres, D., Hsiao, F.-Y., Park, R., Villac, B., and Maruskin, J., "Fundamental Limits on Spacecraft Orbit Uncertainty and Distribution Propagation," AAS Paper 05-471, 2005.

[13] Prussing, J., and Conway, B., Orbital Mechanics, Oxford University Press, Inc., New York, 1993, pp. 3-45. 